\title{
VaR and CVaR Implied in Option Prices
}

\author{
Giovanni Barone Adesi \\ The Swiss Finance Institute at the Università della Svizzera italiana, 6904 Lugano, Switzerland; \\ baroneg@usi.ch; Tel.: +41586664753 \\ Academic Editors: Stefan Mittnik and Marc S. Paolella \\ Received: 16 November 2015; Accepted: 12 February 2016; Published: 29 February 2016
}

\begin{abstract}
VaR (Value at Risk) and CVaR (Conditional Value at Risk) are implied by option prices. Their relationships to option prices are derived initially under the pricing measure. It does not require assumptions about the distribution of portfolio returns. The effects of changes of measure are modest at the short horizons typically used in applications. The computation of $\mathrm{CVaR}$ from option price is very convenient, because this measure is not elicitable, making direct comparisons of statistical inferences from market data problematic.
\end{abstract}

Keywords: VaR; expected shortfall; put option

\section{Introduction}

The successes of the VIX [1] and the SKEW [2] indices of the Chicago Board of Options Exchange show that investors are interested in the information on the distribution of future returns carried by option prices. Although expected volatility and skewness under the objective measure may differ from the two indices, the ease of information available in the option market motivates the success of VIX and SKEW. In fact, option markets provide forward-looking information about the distribution of future asset return that cannot be gleaned using just historical prices.

While the link between option prices and the variance and skewness of the pricing distribution, represented by the two indices, has been analyzed extensively, similar links for the risk measures most popular with financial practitioners and regulators, Value at Risk (VaR) and Conditional Value at Risk (CVaR), are not well known.

$\mathrm{VaR}$ and $\mathrm{CVaR}$ have been objects of many theoretical and empirical investigations. On the theoretical side, the lack of coherence of $\mathrm{VaR}$ played in favor of $\mathrm{CVaR}$ originally. However, the lack of elicitability of the latter [3,4] is likely to re-open the debate. The impossibility of making reliable statistical comparisons about $\mathrm{CVaR}$ estimates from portfolio returns motivates its direct computation from option prices. Option-based $\mathrm{CVaR}$ values can be used as a benchmark to validate alternative methodologies.

Gneiting [3] argues that the lack of elicitability of $\mathrm{CVaR}$ is a likely explanation for the VaR receiving more attention in the empirical literature. See [5,6], for a study of the properties of the filtered historical simulation [7] or alternative estimation methods. Kuester, Mittnik and Paolella [8] provide an extensive comparison of empirical performances of several methods.

For ease of presentation, formulas for the VaR and CVar in terms of options on the underlying portfolio are derived initially under the pricing measure. Later, the effects of measure change are discussed, relating our measures to equivalent results under the objective measure, most commonly used in risk management. Finally, we discuss applications and the implementation of our proposed estimators.

\section{VaR and CVaR}

The Value at Risk, VaR, is the 1- $\alpha$ percentile of the distribution of possible portfolio losses associated with the portfolio value, $\mathrm{S}$, at a given investment horizon, $\mathrm{T}$. That is, VaR is a measure that 
summarizes portfolio losses $\mathrm{L}$, defined as $S_{0}-\mathrm{S}$. $S_{0}$ is a reference value, usually the portfolio value at time 0 [9].

Note that setting $S_{0}$ equal to zero, the probability density of a loss $f(L)$ equals $f(-S)$, that is $\mathrm{F}(\mathrm{L})=1-\mathrm{F}(-\mathrm{S})$. For continuous distributions $\mathrm{VaR} \equiv S_{0}-\mathrm{K}$ such that

$$
\int_{-\infty}^{K} f(S) d S=\mathrm{F}(\mathrm{K})=\alpha
$$

where $\mathrm{F}(\mathrm{K})$ is the distribution function, that is the probability that $\mathrm{S}$ is smaller than $\mathrm{K}$, and $\alpha$ is the chosen confidence level. If the distribution $F(K)$ is not continuous, $K$ is the smallest value for which the integral above equals or exceeds $\alpha$. In either case that is referred as $\operatorname{VaR}(1-\alpha, \mathrm{T})$.

The CVaR, conditional value at risk [10], also known as expected shortfall or conditional tail expectation, is the conditional expected loss within the integration limits in Equation (1):

$$
\mathrm{CVaR}=\frac{1}{\alpha} \int_{-\infty}^{K} L(S) f(S) d S
$$

Although CVaR is popular with theorists, being a subadditive measure, its computation has been hampered by the difficulty of comparing in practice different methods of computing the tail expectation of losses from the unknown distribution generating empirical returns. This difficulty stems from the non-elicitability of CVaR. Therefore, it is very convenient that option prices reveal VaR and CVaR values, independently of the distribution generating them. This will be shown to hold exactly under the pricing measure. It will lead to useful approximations at short horizons under the real world measure.

Equations (1) and (2) are related to the equations that express the European put option price, $p$, and its derivative $\mathrm{x} \equiv \partial \mathrm{p} / \partial \mathrm{K}$, with respect to its strike price $\mathrm{K}$ under the pricing measure

$$
\begin{gathered}
\mathrm{p}=e^{-r T} \int_{0}^{K}(K-S) f(S) d S \\
\mathrm{x}=\frac{\partial p}{\partial k}=e^{-r T} \int_{0}^{K} f(S) d S=e^{-r T} \mathrm{~F}(\mathrm{~K})
\end{gathered}
$$

In Equations (3) and (4) $\mathrm{r}$ refers to the risk-free rate and $\mathrm{T}$ to the time to maturity of the option. As shown in [11], Equations (3) and (4) are valid for any pricing density $f(S)$ under a given probability measure. These results stem from [12], or [13]. In particular, Equation (4) links the pricing distribution function, $F(K)$, to the derivative of a put option with respect to its strike price, $x$. This derivative can be computed analytically or, if an option pricing model is not available, it can be approximated numerically.

If the portfolio has limited liability, the lower limit of integration is zero rather than $-\infty$ in Equations (1) and (2). Comparing then Equations (1) and (4) it follows that, under the pricing measure, the VaR is formally identical to the expression for $\mathrm{x}$ being equal to $\alpha$, except for discounting at the risk-free rate.

The VaR under the risk neutral measure is therefore the difference between the initial portfolio value and the strike price of a European put. The partial derivative of this put with respect to its strike price, $x$, equals the discounted value of $\alpha$, the chosen VaR level at horizon $T$.

To illustrate the above result in terms of the well-known Black-Scholes model [14], the VaR is the difference between the initial portfolio value and the strike price of a put with volatility $\sigma$, for which

$$
\mathrm{N}\left(-d_{2}\right)=1-\mathrm{N}\left(\frac{\ln \left(\frac{S}{K}\right)+\left(r-\frac{\sigma^{2}}{2}\right)}{\sigma \sqrt{T}}\right)=\alpha
$$


Equations (2) and (3) are similarly related. Under limited liability, the lower integration limit in Equation (2) is zero. Writing $S_{0}=K+S_{0}-K$, it follows from Equations (1)-(3) that

$$
\mathrm{CVaR}=e^{r T} p / \alpha+\mathrm{VaR}
$$

\section{Change of Measure}

In the above equations, the same probability measure is used to compute risk measures and option prices. In practice, risk measures are usually computed under the physical, or real world, probability measure. For an extensive discussion on the meanings of the two probability measures in risk management, see [15].

Equations (1) and (2) are usually computed under a different measure than Equations (3) and (4). The probability density $f(S)$ changes to $g(s)$ under the real world measure in those equations. Equations (7) and (8) express VaR under the physical measure, $\mathrm{VaR}=S_{0}-\mathrm{K}^{\prime}$, with

$$
\begin{gathered}
\mathrm{K}^{\prime}: \int_{-\infty}^{K^{\prime}} g(S) d S=\alpha \\
\mathrm{CVaR}=\frac{1}{\alpha} \int_{-\infty}^{K^{\prime}} L(S) g(S) d S
\end{gathered}
$$

The change of measure is simple if $\mathrm{g}(\mathrm{S})$ is known. As an example, in the Black-Scholes model, with risk premium $\mu-r$,

$$
\mathrm{K}^{\prime}=\mathrm{K} e^{(\mu-r) T}
$$

Also, in the computation of the CVaR, $\mathrm{K}^{\prime}$ and $\mu$ replace $\mathrm{K}$ and $\mathrm{r}$ in Equation (5). The change of measure is equivalent to the introduction of a continuous dividend yield $r-\mu$. These results follow because $\ln (\mathrm{S})$ is normal. The two distributions of the logarithms of $g$ and $f$ differ only because of a translation, due to the risk premium. Analogous developments are obtained for other distributions, if the Girsanov theorem holds. Otherwise, the change can be computed starting from the ratio of the two densities, $g$ and $f$.

In the more general case, the relationship between $\mathrm{g}, \mathrm{f}, \mathrm{K}$ and $\mathrm{K}^{\prime}$ is determined by the pricing kernel, that is the discounted ratio of $\mathrm{f}$ and $\mathrm{g}$. However, the pricing kernel is generally not given and its estimation is problematic. It is always true that if $g(S)$ is smaller than $f(S)$ for the values of $S$ on the left tail of the distribution, as it is usually assumed to be the case for market indices, $\mathrm{K}$ is on the left of $\mathrm{K}^{\prime}$. Moreover, if the stochastic discount rate associated to each state tends to zero when $\mathrm{T} \rightarrow 0$, that is the gross stochastic discount rate tends to one, the limit of $f$ is $g$ and the limit of $K$ is $K^{\prime}$.

That follows from the fact that the state price is the present value of its pricing probability discounted at the risk-free rate over the time horizon or, equivalently, its real world probability discounted at the risk-free discount rate. When the horizon goes to zero, it follows that $f$ converges to $\mathrm{g}$, because both of them approach the state price density. This condition, equivalent to requiring that the rate of return exists in each state when the time horizon approaches zero, ensures that option prices provide a valid approximation of VaR and CVaR, computed at short horizons under the real world measure. Therefore, as shown in the following section, we do not need to rely on a specific change of measure, as in [16], to provide a useful approximation of VaR and CVaR.

\section{Extensions and Applications}

Some other risk measures can be easily derived along the same principles. As an example, a measure of tail asymmetry is

$$
\operatorname{CVaR}\left[S, \alpha, K_{\alpha}(S)\right]-\operatorname{CVaR}\left[-S, \alpha, K_{\alpha}\left(-S,-S_{0}\right)\right]
$$


where $K=\alpha$ is the value of $K$ associated with the confidence level $\alpha$. The second term in Equation (10) equals the value of a call on $S$ with strike price $K_{1-\alpha}(S)$ such that $F\left(K_{1-\alpha}(S)\right)$ equals $1-\alpha$, compounded at the risk-free rate. It is now necessary to use a call rather than a put, because the lower integration bound, introduced in Equation (1), can be no longer truncated at zero.

The measure in Equation (10) is zero for symmetric distributions and positive for distributions with heavier left tail.

Our measures of risk rely on option prices. Their limitation is that option prices are easily available for stock indices and some exchange-traded funds (ETFs), not on generic portfolios. Moreover, their time to maturity does not always equal the desired risk horizon. However, similar restrictions do not impede the use of VIX and SKEW, because most professionally managed portfolios are highly correlated with the index underlying these variance and skewness indicators and maturities can be easily interpolated. Similar developments for $\mathrm{VaR}$ and $\mathrm{CVaR}$, relating two risk measures to option prices, may be useful within the financial industry.

An advantage of our measures is that they rely on one single option, rather than the summation of infinite options. This ensures that they are not affected by the changing truncation errors afflicting VIX and SKEW, which in theory are based on infinite sums of options. The reliance on a single option price makes our measures also very useful to validate risk models, that should come close to matching the values derived from option prices over short horizons.

To implement the above equations it is necessary to identify $K_{\alpha}$. This task can be accomplished starting from model option prices or, without relying on a specific option-pricing model, from numerical differentiation of contiguous market prices.

Let $\mathrm{R}=\frac{p_{2}-p_{1}}{K_{2}-K_{1}}$ be the incremental ratio of the put price over the strike price of two puts with contiguous strike prices, $K_{2}>K_{1}$. The limit of of $\mathrm{R}$ when $K_{2} \rightarrow K_{1}$ is $\mathrm{x}$, that is $\alpha$ (Equation (4)).

For finite increments, being $x$ monotonically increasing in $K, R$ overstates $x$ at the left point, $K_{1}$, and understates it at the right point. To remedy this, it is convenient to use three options with contiguous strike prices, averaging the value of $\mathrm{R}$ from the first two with the value from the last two options. The average is an estimate of $x$ at the middle point, $K_{2}$, that eliminates the first-order error in a Taylor expansion of the derivative. This procedure eliminates also the first-order error due to the implied volatility changing across strike prices [15], simplifying the procedure proposed in [16].

As an example, consider three puts with strike prices $24,25,26$, on an asset with price 35 . The risk-free rate is $6 \%$ and their time to maturity, $T$, is 0.5 . Their market prices are $0.063,0.106$ and 0.168. To estimate $x(K=25)$, compute the ratios of differences of option prices to the left and to the right of this point:

$$
\frac{0.106-0.063}{25-24}=0.0425 \frac{0.168-0.106}{26-25}=0.062
$$

The average of the two differences, 0.052 , compounded by the negative exponential of $0.06 \times 0.5=0.03$, is 0.054 . This is the value of the confidence level of the VaR associated with $p(25)$. The VaR at $5.4 \%$ for a six-month horizon under the risk neutral measure is therefore $35-25=10$. From Equation (6), the corresponding CVaR is

$$
\mathrm{CVaR}=e^{0.03} \times 0.106 / 0.054+10=12.02
$$

The put prices in our example are the Black-Scholes prices for volatility $\sigma=0.30$. Our choice of theoretical, rather than market prices, is due to the opportunity they offer to compute exact values for risk measures, allowing for a comparison with our approximations. That would not be possible for CVaR, which is not elicitable.

The value of $\mathrm{N}(-\mathrm{d} 2)$ with the above parameters is 0.0525 rather than the approximated value, 0.05 . The correct value may be approximated better with more sophisticated interpolation methods. Of course, the exact confidence level is known only if option prices follow Black-Scholes or another specific model. 
If the Black-Scholes model holds, it is possible to compute VaR and CVar under the real world measure if a risk premium, as an example $\mu-r=0.08$, is assumed. The VaR at $5.4 \%$ level and 6-month horizon becomes under the real world measure

$$
\mathrm{VaR}=35-25 e^{0.08 \times 0.5}=8.98
$$

The corresponding CVaR can be computed substituting $\mathrm{p}(25)=0.106$ with $\mathrm{p}\left(25 e^{0.08 \times 0.5}\right)$ in Equation (12). In the computation of the latter put option, the drift is the risk free rate increased by the risk premium that acts as a negative dividend, because the distribution of the portfolio value under the change of measure changes only its drift. The $\mathrm{CVaR}$ at $5.4 \%$ under the physical measure is therefore

$$
\mathrm{CVaR}=8.98+\frac{e^{r T}}{\alpha}\left[\mathrm{p}\left(\mathrm{S}, K^{\prime}, \mathrm{T}, \sigma, \mathrm{r}, \mathrm{d}\right)=\mathrm{p}(35,26.02,0.5,0.3,0.06,-0.08)\right]=11.06
$$

The numerical values in Equations (12) and (14) show that the change in CVaR, due to the change of measure, is smaller than the change in VaR. This result applies to the Black-Scholes model, but it is not true in general. However, because it is always true that the difference between the two VaR, or the CVaR values, under the two measures always approaches zero for smaller times to maturity, option prices provide a useful approximation to $\mathrm{VaR}$ and $\mathrm{CVaR}$ at short time horizons.

Readers can verify that, if the six-month horizon, chosen in our example, were to be replaced by the ten-day horizon often used in applications, the percentage difference between the two risk measures would decrease almost proportionally, that is to about one per cent in our example. This stems directly from the discounted expectations under either measure converging to the same state price when the time horizon $\mathrm{T}$ approaches zero. Therefore, their difference goes to zero as $\mathrm{cT}$, where $\mathrm{c}$ is the average risk premium associated with the relevant states.

\section{Conclusions}

Option prices provide information about the distribution of potential losses in portfolio returns. The derivative of a put option price with respect to its strike price allows for the immediate computation of $\mathrm{VaR}$ and $\mathrm{CVaR}$ under the pricing measure. This derivative can be approximated from the differences in market prices of puts and their strike prices.

For the lognormal distribution, the VaR is more sensitive than the CVaR to the change in measure. For any distribution, the dependence of both $\mathrm{VaR}$ and CVaR from the change of measure vanishes at short maturities, where option prices provide good approximations to VaR and CVaR under the real world probability measure. This result is most useful because CVaR is not an elicitable measure, making statistical inferences from portfolio returns problematic.

Acknowledgments: This research has been supported by The Swiss Finance Institute and the Swiss National Fund Grant 153135. Referees' comments are gratefully acknowledged. I am solely responsible for remaining errors.

Conflicts of Interest: The author declares no conflict of interest.s

\section{References}

1. Whaley, R.E. Understanding the VIX. J. Portf. Manag. 2009, 35, 98-105. [CrossRef]

2. Chicago Board Options Exchange (CBOE). THE CBOE Skew Index; Monograph; CBOE: Chicago, 2010.

3. Gneiting, T. Making and Evaluating Point Forecasts. J. Am. Stat. Assoc. 2011, 106, 746-762. [CrossRef]

4. Ziegel, J.F. Coherence and Elicitability. Math. Financ. [Online early access]. [CrossRef]

5. Gao, F.; Song, F. Estimation Risk in GARCH VaR and ES Estimates. Econom. Theory 2008, 24, $1404-1424$. [CrossRef]

6. Taylor, J.W. Estimating Value at Risk and Expected Shortfall Using Expectiles. J. Financ. Econom. 2008, 6, 231-252. [CrossRef]

7. Barone-Adesi, G.; Giannopoulos, K.; Vosper, L. Backtesting Derivative Portfolios with Filtered Historical Simulation (FHS). Eur. Financ. Manag. 2002, 8, 31-58. [CrossRef] 
8. Kuester, K.; Mittnik, S.; Paolella, M.S. Value-at-Risk Prediction: A Comparison of Alternative Strategies. J. Financ. Econom. 2006, 4, 53-89. [CrossRef]

9. Jorion, P. Value at Risk: The New Benchmark for Managing Financial Risk, 3rd ed.; McGraw-Hill: New York, NY, USA, 2006.

10. Embrechts, P.; Kluppelberg, C.; Mikosch, T. Modelling Extremal Events for Insurance and Finance; Springer: New York, NY, USA, 1997.

11. Mitra, S. The Relationship between conditional value at risk and option prices with a close form solution. Eur. J. Financ. 2015, 21, 400-425. [CrossRef]

12. Breeden, D.; Litzenberger, R. Prices of State-Contingent Claims Implicit in Option Prices. J. Bus. 1978, 51, 621-651. [CrossRef]

13. Ingersoll, J.E. Theory of Financial Decision Making; Rowman and Littlefield: Lanham, MD, USA, 1987.

14. Black, F.; Scholes, M. The Pricing of Options and Corporate Liabilities. J. Political Econ. 1973, 81, 637-659. [CrossRef]

15. Aït-Sahalia, Y.; Lo, A.W. Non Parametric Risk Management and Implied Risk Aversion. J. Econom. 2000, 94, 9-51. [CrossRef]

16. Barone-Adesi, G.; Elliott, R. Cutting the Hedge. Comput. Econ. 2007, 29, 151-158. [CrossRef]

(c) 2016 by the author; licensee MDPI, Basel, Switzerland. This article is an open access article distributed under the terms and conditions of the Creative Commons by Attribution (CC-BY) license (http://creativecommons.org/licenses/by/4.0/). 